\title{
The silicomolybdic acid spectrophotometric method and its application to silicate/biopolymer interaction studies
}

\author{
Thibaud Coradin ${ }^{\mathrm{a}, *}$, David Eglin ${ }^{\mathrm{a}, \mathrm{b}}$ and Jacques Livage $\mathrm{e}^{\mathrm{a}, \mathrm{b}}$ \\ ${ }^{a}$ Laboratoire de Chimie de la Matière Condensée, CNRS-UMR 7574, Université Pierre et Marie Curie, \\ 4 place Jussieu, 75252 Paris cedex 05, France \\ ${ }^{\mathrm{b}}$ Collège de France, 11 place Marcelin Berthelot, 75005 Paris, France
}

\begin{abstract}
The possibility to understand natural strategies to build-up silica $\mathrm{SiO}_{2}$ networks relies on the investigations of the kinetics of silica precursors polymerization in the presence of biopolymers. The silicomolybdic acid spectrophotometric method allows to study this process at the molecular level, provided that reliable procedures are available. Moreover, special care must be taken to avoid interfering processes induced by the inorganic and/or bio-organic species found in solution. In order to illustrate the possibilities and limitations of this method, different sets of data are presented and discussed in the context of silica formation control by natural systems.
\end{abstract}

\section{Introduction}

Biominerals deposited by living organisms have become paradigms for the elaboration of new materials with controlled morphology and properties [1]. This "bio-inspired" strategy relies on the possibility to understand the biological routes to mineral formation. A common approach involves the extraction and analysis of proteins, or polysaccharides, associated with the inorganic material within the organisms, which are likely to take part in the biomineralization process [2,3]. However, because of the diversity and complexity of isolated molecules, a "biomimetic" approach using model systems can also provide interesting hints on the mechanisms of solid growth control by biological species [4-6].

Among the most widespread biogenic minerals, silica $\mathrm{SiO}_{2}$ exhibits several rather unique features [7]. In contrast to common calcium carbonate or phosphate, its formation does not proceed via precipitation of ions but involves an inorganic polymerization process from molecular precursors [8]. Thus, amorphous solids are obtained, which can accommodate a large variety of shapes, as illustrated by the finely structured shells of diatoms [9]. Moreover, the development of sol-gel chemistry has allowed to synthesize silica in experimental conditions compatible with proteins and cells [10].

Numerous methods are available to characterize biogenic or bio-inspired/bio-mimetic silica: ${ }^{29} \mathrm{Si}$ solid state Nuclear Magnetic Resonance (NMR), infrared spectroscopy, electron microscopy, physisorption porosimetry, ... [11]. However, the dynamic of the polymerization processes is much more difficult to

\footnotetext{
${ }^{*}$ Corresponding author: Dr. Thibaud Coradin, Laboratoire de Chimie de la Matière Condensée, Université Pierre et Marie Curie, 4 place Jussieu, 75252 Paris cedex 05, France. Tel.: +33 1442755 17; Fax: +33 1442747 69; E-mail: coradin@ @cr. jussieu.fr.
} 
monitor. ${ }^{29} \mathrm{Si}$ solution NMR studies should, in principle, give access to these informations but, because of the low natural abundance of the NMR-active ${ }^{29} \mathrm{Si}$ isotope, high concentrations of silica have to be used, which do not reflect the diluted state of silica precursors in natural systems. Photon Correlation Spectroscopy may also be used to follow silica particle growth during the mineral formation but it is limited to the latter steps of the reaction where colloidal species are already formed. In contrast, the silicomolybdic acid (hereafter denoted SMA) method, which allows to titrate monosilicic acid $\mathrm{Si}(\mathrm{OH})_{4}$, the natural precursor of $\mathrm{SiO}_{2}$ in aqueous environments, provides a powerful tool to study the polymerization process from its very beginning [12].

As a matter of fact, the principle of SMA assays was reported more than a century ago [13]. Over the years, a better understanding of the chemical processes involved in this method was achieved and numerous experimental procedures were reported taking into account the specificity of each media [14-20]. Further technical progresses were also possible, leading to the commercialisation of automatic silica analyzers (Aquacon Si, IOTRONIC; Series 5000 Silica Analyzer, HACH). Nevertheless, because of the large amount of literature available, it is sometimes difficult to find simple, reliable experimental procedures to perform SMA assays.

In this paper, we tried to gather most significant information from the literature and present simple procedures for the silicomolydic acid measurements. In a second part, applications of the SMA method to investigate silicate/biopolymer interactions in the context of biomimetic studies are described. Finally, the possibilities and limitations of this method are discussed.

\section{The silicomolybdic acid spectrophotometric method}

\subsection{From silicates to silica}

In diluted aqueous solutions, soluble silica is found as monosilicic acid $\mathrm{Si}(\mathrm{OH})_{4}$. This monomer has a $\mathrm{pK}_{\mathrm{a}}$ of about 9 and thus also exist in the deprotonated $\mathrm{SiO}(\mathrm{OH})_{3}^{-}$form above $\mathrm{pH}$ 8. Increasing the concentration leads to condensation of two monomers, following Eq. (1)

$$
(\mathrm{OH})_{3} \mathrm{Si}-\mathrm{OH}+\mathrm{HO}-\mathrm{Si}(\mathrm{OH})_{3} \Leftrightarrow(\mathrm{OH})_{3} \mathrm{Si}-\mathrm{O}-\mathrm{Si}(\mathrm{OH})_{3}+\mathrm{H}_{2} \mathrm{O} .
$$

Further steps involve the formation of trimers, tetramers and then higher oligomeric species until $1-2 \mathrm{~nm}$ silica nanoparticles are formed. In the meantime, the $\mathrm{pK}_{\mathrm{a}}$ of the polysilicic acids decreases down to 6.5 so that negatively charged silicate species become predominant above $\mathrm{pH} 7-8$. In these conditions, colloidal species aggregation is prevented by electrostatic repulsive interactions so that further particle growth occurs via the Ostwald ripening process, leading to stable sols. In contrast, below $\mathrm{pH} 7$, particles are only slightly charged so that they tend to aggregate, finally forming gels. Addition of salts can shield the electrical charge of the colloids and induces precipitation. Polymers may also interact with particle surface, inducing either coagulation via charge shielding and interparticle bridging or stabilization of the sol via steric hindrance [12].

It therefore appears that the latter steps of silica formation from aqueous precursors can be studied using the wide range of technical approaches developed for colloidal systems analyses [21]. However, getting information on the very first steps of the condensation process relies on the possibility to study small silicate species, and especially, the monomeric silicic acid $\mathrm{Si}(\mathrm{OH})_{4}$. 


\subsection{Presentation of the method}

The silicomolybdic acid (SMA) spectrophotometric method is based on the ability of silicic acid to form a heteropolyacid, silico-12-molybdic acid, in the presence of acidified ammonium heptamolybdate, following Eq. (2)

$$
7 \mathrm{Si}(\mathrm{OH})_{4}+12 \mathrm{H}_{6} \mathrm{Mo}_{7} \mathrm{O}_{24} \cdot 4 \mathrm{H}_{2} \mathrm{O}+17 \mathrm{H}_{2} \mathrm{O} \Leftrightarrow 7 \mathrm{H}_{4} \mathrm{SiMo}_{12} \mathrm{O}_{40} \cdot 29 \mathrm{H}_{2} \mathrm{O} .
$$

The resulting silicomolybdic acid is a yellow compound which can be titrated spectrophotometrically by monitoring the optical density at $\lambda=400 \pm 10 \mathrm{~nm}$.

As a matter of fact, the silicomolybdic acid exists in two isomeric forms, $\alpha$ - and $\beta$-, the $\beta$-form exhibiting an extinction coefficient twice as large as the $\alpha$-form in the visible region [14]. The critical factor determining the formed isomer was first reported to be the silicic acid: molybdate ratio, but further studies showed that $\mathrm{pH}$ was actually the crucial parameter. The $\alpha$-form is obtained between 3.8 and 4.8 whereas the $\beta$-isomer is the more stable between $\mathrm{pH} 1.0$ and $\mathrm{pH} 1.8$ [18].

The silicomolybdic acid is a cage-like structure where the silicon atom is in a tetrahedral cavity of oxygen atoms belonging to four of the twelve $\mathrm{MoO}_{6}$ octahedra delimiting the heteropolyacid [22] (Fig. 1). Thus, only monomeric silicic acid can, in principle, yield to the yellow compound formation. However, this method is mainly applied for dynamic studies of silicate solutions where polymerization/depolymerization can take place, so that silicic acid may still be consumed/formed during the SMA measurements. Depolymerization might also occur because experimental procedures require a dilution step of the investigated sample in order to reach concentration ranges compatible with its spectrophotometric detection. Such a polymerization/depolymerization process occurring simultaneously with the optical measurement would lead to non-stable optical density measurements. Hopefully, the method is carried out at low $\mathrm{pH}$ where condensation/hydrolysis reactions are slow so that the acidification step of the SMA method mainly freezes the reaction media. In this case, only monomeric silica initially present in the investigated solutions, as well as oligomers that are small enough to be fully depolymerised during the delay between acidified molybdate addition and spectrophotometric measurements, are titrated. As a matter of fact, the reaction rate of silicates with the molybdate reagent may give an indication of their degree of oligomerization $[8,12,23]$.

The yellow silicomolybdic method nevertheless exhibits several limitations. First of all, its molar extinction coefficient at $\lambda=400 \mathrm{~nm}$ is $\varepsilon=1500 \pm 20 \mathrm{lmol}^{-1} \mathrm{~cm}^{-1}$ so that the limit of detection is in the

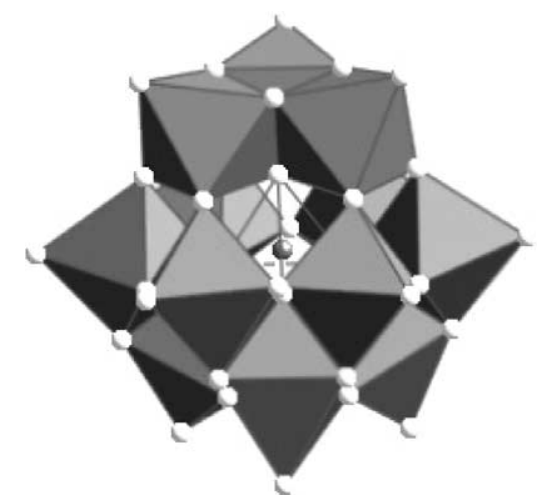

Fig. 1. Structure of the silicomolybdic acid cluster. The silicon atom (in gray) is encaged by twelve $\mathrm{MoO}_{6}$ octahedra (oxygen atoms in white). Hydrogen atoms are not shown for convenience. 


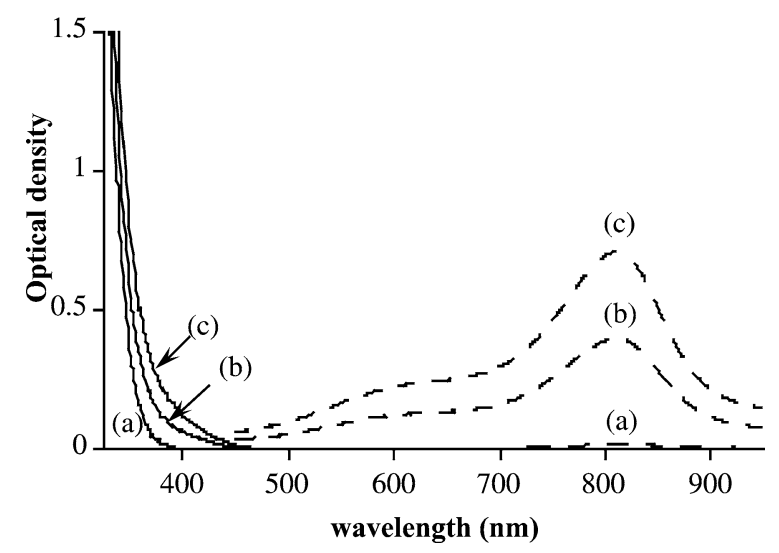

Fig. 2. UV-visible spectra of silicic acid solutions at concentrations of (a) 0 ppm, (b) 5 ppm and (c) $10 \mathrm{ppm}$, as titrated with the yellow SMA method (plain line) and the blue SMA method (dashed line).

order of $10^{-4} \mathrm{moll}^{-1}$ (Fig. 2). Another critical issue is the possibility for phosphate ions, widely present in natural samples or experimental buffers, to form a yellow phosphomolybdic polyacid strongly absorbing at the same wavelength [24]. The first problem was solved by taking advantage of the possibility to reduce the silicomolybdic acid to the so-called molybdenum blue complex, exhibiting an absorption maximum at $\lambda=810 \mathrm{~nm}$ with a molar extinction coefficient $\varepsilon=44700 \pm 150 \mathrm{lmol}^{-1} \mathrm{~cm}^{-1}$ so that concentrations as low as $5 \times 10^{-6} \mathrm{moll}^{-1}$ can be accurately measured [25] (Fig. 2). Different reducing agents were investigated including tin(II) chloride, ascorbic acid, 1-amino-2-naphtol-4-sulphonic acid and 4-methylaminophenol sulphate (metol) in the presence of sodium sulphite [16,26]. A survey of the literature indicates that both $\mathrm{SnCl}_{2}$ and the metol-sulphite system are still used [27,28], the former reacting more rapidly (less than 5 minutes) but requiring to be kept away from air oxidation. Moreover, the reduction of the unreacted molybdate reagent, which would contribute to the measured optical density, must be prevented by increasing the solution acidity via sulphuric acid addition. As far as phosphate ions are concerned, it was shown that adding oxalic acid leads to the phosphomolybdic complex breakdown [29].

\subsection{Experimental procedures}

Different procedures can be found in the literature, which mainly differ in sample preparation (dissolution, dilution), reaction time and, in the case of the molybdenum blue method, on the reducing agent. Hereafter are proposed procedures whose reliability was checked by repeated calibration assays using silicate standard solutions (1000 $\left.\mathrm{ppm} \mathrm{SiO}_{2}, \mathrm{BDH}\right)$ and used in some of the silicate/biopolymer investigations described below.

\subsubsection{The yellow silicomolybdic acid assay}

In a typical experiment, $1 \mathrm{ml}$ of the silicic acid solution is sampled and diluted to $12.5 \mathrm{ml}$ with deionized water. $500 \mu \mathrm{l}$ of a $100 \mathrm{gl}^{-1}$ ammonium molybdate tetrahydrate $\left(\mathrm{NH}_{4}\right)_{6} \mathrm{Mo}_{7} \mathrm{O}_{24} \cdot 4 \mathrm{H}_{2} \mathrm{O}$ aqueous solution and $500 \mu \mathrm{l}$ of a $1.5 \mathrm{M}$ sulfuric acid aqueous solution are then added under stirring. The yellow colour is left to develop over $10 \mathrm{~min}$ at room temperature before measuring the optical density at $\lambda=400 \mathrm{~nm}$.

Within this reaction time, only dimeric silicic acid can be hydrolyzed to form titrable monosilicic acid. Longer reaction times would allow larger oligomers to be depolymerized so that a 10 min delay should 
be strictly respected. This procedure gives a linear calibration curve in the $10^{-2}-10^{-3}$ moll $1^{-1}$ silicic acid concentration range. For higher concentrations, the dilution volume can be increased. Smaller sampling volumes, down to $200 \mu \mathrm{l}$, can also be used, provided that the other volumes are proportionally scaled down. Moreover, after molybdate and sulfuric acid solutions addition, stirring time is limited to 5 min, followed by a $5 \mathrm{~min}$ resting period allowing possible large diffusing silicate species to settle down. Note that the molybdate solutions are only stable for 2 to 3 weeks at room temperature. They are better stored at $4{ }^{\circ} \mathrm{C}$ in the dark and warmed up before use. In any case, new solutions must be regularly prepared.

\subsubsection{The blue silicomolybdic assay}

Solution A is made of $20 \mathrm{gl}^{-1}$ ammonium molybdate tetrahydrate and $60 \mathrm{mll}^{-1}$ concentrated hydrochloric acid in deionized water. Solution B is prepared by adding oxalic acid $(20 \mathrm{~g})$, 4-methylaminophenol sulphate (6.67 g), anhydrous sodium sulfite $(4 \mathrm{~g})$, deionised water $(500 \mathrm{ml})$ and concentrated sulphuric acid $(100 \mathrm{ml})$ in a 11 volumetric flask and completed with deionised water.

In a typical experiment, $1 \mathrm{ml}$ of the unknown concentration silicic acid solution is sampled and diluted to $16 \mathrm{ml}$ with deionised water. To this is added $1.5 \mathrm{ml}$ of solution A. After 10 minutes, $7.5 \mathrm{ml}$ of solution $\mathrm{B}$ is added to the assay solution. The blue colour is left to develop over 2 hours at room temperature before measuring the optical density at $\lambda=810 \mathrm{~nm}$.

As for the yellow SMA method, only monomeric and dimeric silicic acids are titrated provided that first 10 min delay is respected. Then, the blue colour obtained from the reduction step remains stable over 24 hours. This procedure gives a linear calibration in the $5 \times 10^{-4}-5 \times 10^{-5} \mathrm{moll}^{-1}$ silicic acid concentration range. Preceeding remarks on dilution or sampling volume variation also apply here.

The SMA reproductibility has been checked by several authors and readings on a series of standards showed a relative standard deviation for the yellow SMA at $5.0 \times 10^{-3} \mathrm{moll}^{-1}$ of $\pm 2 \%$ and for the blue SMA at $0.083 \times 10^{-3} \mathrm{moll}^{-1}$ of $\pm 1 \%$. In the presence of inorganic ions and organic molecules, it is often that standard deviations increase due to interferences with SMA (vide infra).

\subsection{Interfering species}

The possible interference of various inorganic ions has already been thoroughly investigated and will not be discussed here. It is nevertheless interesting to point out that among species which are more likely to be associated with silica in biogenic samples, $\mathrm{Al}(\mathrm{III})$ does not interfere with silica titration whereas $\mathrm{Fe}(\mathrm{III})$ ions lead to the precipitation of ferric molybdate, this problem being overcome by reduction to $\mathrm{Fe}(\mathrm{II})$ using hydroxylamine [16]. As far as phosphate anions are concerned, it was mentioned above that they are able to form a yellow phosphomolybdate complex. Thus, even though the addition of oxalic acid can prevent the interfering effect, phosphate buffers should be avoided and can be conveniently substituted by organic buffers such as Tris.

In the case of organics, three types of interference may arise. The possibility for sugars and hydroxylated polymers [12], as well as gelatine, to interact with the molybdate reagent can prevent the formation of the silicomolybdic complex and even induces the appearance of a yellow colour in the absence of silica. Alternatively, the SMA can remain adsorbed at the surface of collagen, requiring the separation of the polymer from the solution via centrifugation. Finally, the presence of diffusing species, such as vesicles, which cannot be easily separated from the analysis media may contribute to the optical density at $\lambda=400 \mathrm{~nm}$ for the yellow silicomolybdic complex. This may be overcome by conversion to the molybdenum blue compound whose characteristic wavelength is high enough to significantly reduce the diffusion contribution. 


\section{Application to silicate/biopolymers interaction studies}

\subsection{Biogenic silica formation studies}

A large number of natural systems, including bacteria, algae, plants and insects, are able to extract silica precursors from their environment and to transport them to specific deposition sites where condensation occurs. In addition, they are able to control the morphology of the deposited mineral phase, as illustrated by the highly aesthetic silica shells of radiolarian and diatoms [7].

In common with other biomineralization processes, this building-up of silica network is controlled by biomolecules (proteins, polysaccharides, glycoproteins,...). Some of these were identified in certain plants, sponges and diatoms and their ability to activate silica formation and to achieve a partial morphological control was already reported [30]. However, because of the diversity and complexity of these systems, it was proposed to follow a step-by-step biomimetic approach, involving the use of well-known biomolecules of increasing complexity in order to understand how they interact with silica precursors at different complexity levels.

One of the major issues of these studies is the choice of the silica precursors source. Materials chemists have been using silicon alkoxides $\mathrm{Si}(\mathrm{OR})_{4}$ for many years but these compounds should undertake an hydrolysis step to form $\mathrm{Si}-\mathrm{OH}$ groups, releasing the $\mathrm{ROH}$ parent alcohol which may not be compatible with biological species [31]. Colloidal silica can also be used but it is known that it cannot be extracted as such from the environment by the living organisms so that it is only relevant for studying latter steps of the silica building-up process [32]. In contrast, the possibility for natural systems to stabilize silica precursors during their transport through chelation opened the way for a series of investigation of different $\mathrm{Si}$ (cathecolate) ${ }_{3}$ complexes [33]. Alternatively, sodium silicate aqueous solutions, containing naturally-occurring silicic acids, appear as suitable sources for these biomimetic studies, both because they are biologically relevant and conveniently studied by the SMA method.

\subsection{Behaviour of the sodium silicate solutions}

In a first step, it is important to check the behaviour of initial silica solutions via the SMA method. This behaviour mainly depends on the solution concentration and reaction $\mathrm{pH}$ [34]. This is illustrated in Fig. 3 where $30 \mathrm{ml}$ of a $10 \mathrm{mM}$ solution of sodium silicate was obtained by dilution of the initial $\mathrm{NaOH} 10 \%: \mathrm{SiO}_{2} 27 \%$ aqueous solutions in different buffers and mixing for 15 minutes before $3 \mathrm{ml}$

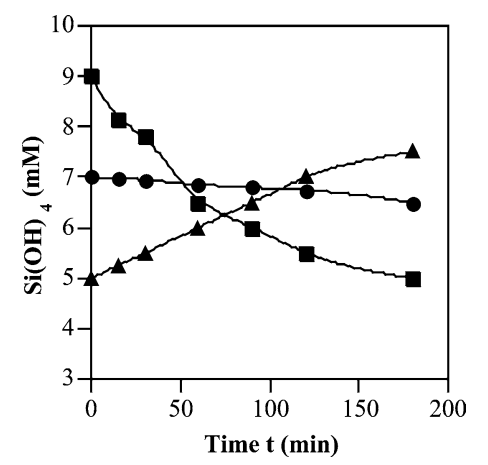

Fig. 3. Evolution with time of the concentration of silicate species titrable by the yellow silicomolybdate method for a $10 \mathrm{mM}$ sodium silicate solution at $\mathrm{pH} 4.7$ (triangle), $\mathrm{pH} 7.2$ (circle) and $\mathrm{pH} 9.2$ (square). 
additional buffer was added and the measurements started using the yellow SMA method. Note first that the starting values of detectable silicate species increase with $\mathrm{pH}$. As a matter of fact, the initial concentrated silicate solution contains a mixture of oligomers that tend to depolymerize upon dilution, this process being all the faster as the $\mathrm{pH}$ increases. However, the formed monomeric or dimeric species can also undertake condensation and the rate of this reaction also increases with $\mathrm{pH}$. What is observed

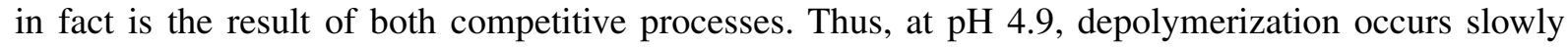
but condensation is also limited, resulting in an increase in the amount of titrable species with time. In contrast, at $\mathrm{pH}$ 9.2, depolymerization is fast but formed titrable species also condense quickly, leading to a rapid decay of the measured SMA. The intermediate $\mathrm{pH} 7.2$ value also leads to a global decrease in monomeric and dimeric silicic acid, but it is far more limited than at $\mathrm{pH} \mathrm{9.} \mathrm{In} \mathrm{addition} \mathrm{to} \mathrm{these}$ informations on the silicate kinetics behaviour, these data also indicate that these solutions, even after dilution, do not contain only monomeric silicic acid but also higher oligomers, which may react in a different way with added biomolecules. However, the SMA method does not provide any information on the size and relative amount of these oligomers, pointing out the main limitation of this technic.

\subsection{Effect of biomolecule addition on sodium silicate solutions}

When biomolecules diluted in the $3 \mathrm{ml}$ buffer were added to the $10 \mathrm{mM}$ silicate solution at $\mathrm{pH} 7.2$, different kinds of yellow SMA curves could be obtained (Fig. 4). First, adding different amino acids (proline, serine, lysine, arginine) did not noticeably modify the kinetics measurements, suggesting that no important interactions arise between silicates and these molecules [34]. Then, upon addition of DNA, alginic acid, bovine serum albumin and poly-serine [34-36], a noticeable decrease in the amount of titrable species with time is observed but the initial solution remains clear and no solid is formed. In these cases, added biomolecules are either negatively charged or neutral so that no strong electrostatic interactions with silicates are expected. Nevertheless, hydrogen bond formation can occur, presumably accounting for the observed change. In this case, the decrease of the SMA measure could either indicate monomer and dimer condensation or their interaction with the polymer, preventing the complex formation. In contrast, poly-(lysine $)_{n}$ and poly-(arginine $)_{m}$ lead to the slow precipitation of a white solid whereas SMA data suggest a continuous and important decrease in monomeric silicic acid $[37,38]$. Interestingly, the kinetics of the decay increase with the polymer chain length, even though the $\mathrm{SiO}_{2}$ : lysine (or arginine) molar ratio was kept constant. Based on an analogy with surfactant/polyelectrolyte systems,

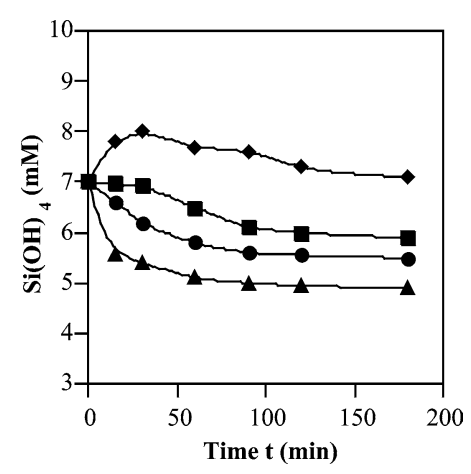

Fig. 4. Evolution with time of the concentration of silicate species titrable by the yellow silicomolybdate method for a $10 \mathrm{mM}$ sodium silicate solution at pH 7.2 in the presence of alginic acid (square), poly-(lysine) 155 (circle), poly-(lysine) 1005 (triangle) and lysozyme (diamond). 
it was suggested that electrostatic interactions between silicate and ammonium groups of the poly-amino acid chain allow the inorganic species to get close one to another, favouring their condensation. Formed oligomeric species could then serve as nuclei for further silica formation. As a matter of fact, when poly-(lysine) ${ }_{1005}$ was used, instant precipitation was observed, reflected by a sudden decrease in SMA measurements, followed by a plateau [38]. This was also observed when poly-proline which exhibit a low solubility, was used [34]. This suggests that, in these cases, the association of the biomolecules with silicate decrease their solubility to a point where they are depleted from the solution. In contrast to small poly-(lysine) chains, the amount of consumed titrable species was proportional to the amount of added polymer, indicating that this process does not involve the activation of silicate condensation but rather the association of isolated silicate species with the biomolecules.

It appears from these examples that SMA measurements are usually in agreement with visual observations of the changes occurring in the solutions. This is however not always true, as illustrated for lysozyme addition [36]. At pH 7.2, the slow formation of a solid suggested a behaviour similar to short poly-(lysine) chains. Surprisingly, the SMA curves showed a first increase in the amount of titrable species and then a slow decrease. This can be understood if one takes into account the presence of undetectable silicate oligomers in solution. Their negative charge increases with their degree of oligomerization, and the larger ones are more reactive towards positively charged species than monomeric or dimeric silicic acids. Lysozyme, from its side, does not exhibit as much lysine or arginine groups as the corresponding homo polyaminoacids so that its positive charge is limited. Therefore, it can be suggested that at the beginning of the reaction, only those larger oligomers are able to interact with the polymer, inducing silica formation. As a consequence, the concentration of free silicates in solution decreases, favouring depolymerization. However, as indicated above, these monomers or dimers have a strong tendency to condense near neutral $\mathrm{pH}$ so that, above a critical value, the amount of titrable species will also start to decrease.

\subsection{Silicon complexes and the molybdenum blue method}

Thus, because of the presence of silicate oligomers in the starting solutions, data analysis is not always straightforward. Indeed, it would be interesting to start from monomeric silicic acid solution but, as indicated above, this may only be achieved at very low concentration. However, it is possible to overcome this problem by using silicon complexes such as $\mathrm{Si}$ (catecholate) ${ }_{3}$ [33]. These compounds can be hydrolyzed at the beginning of the reaction, triggering the release of $\mathrm{Si}(\mathrm{OH})_{4}$. This approach was used to investigate the effect of cellulose [39] as well as biomolecules extracted from the plant Equisetum telmateia $[40,41]$.

Using the molybdenum blue method, it was possible to study in great details the kinetics of the very first steps of the reaction, i.e. mono silicic acid to disilicic acid, disilicic acid to trisilicic acid and trimer to larger oligomers. Because the blue SMA method procedure induces the breakdown of the silicon complexes, ${ }^{1} \mathrm{H}$ NMR was also used to distinguished between Si(catecholate) ${ }_{3}$ and titrable silicic acids [33].

When investigating the interactions of these solutions with cellulose, it was shown that this polymer did not modify neither the complex breakdown neither the first steps of silicic acid condensation kinetics. However, cellulose does modify the size of obtained particles, as well as their aggregation [39]. Similarly to what was suggested for the role of alginic acid and poly-serine on sodium silicate solutions, hydrogen bond formation does not allow the activation of silica condensation but partially controls the behaviour of oligomeric particles. In contrast, the addition of Equisetum telmateia extracts, containing both proteins and glycoproteins, increases the rate of the trimer formation and leads to the deposit of a crystalline 
quartz-like silica structure [40,41]. The role of these proteins on silica condensation activation from silicon complexes is, once again, very similar to the conclusions drawn from sodium silicate solutions.

\section{Conclusion}

Understanding the effect of biomolecules on the condensation of silica precursors is a key-point to unravel the biosilicification processes performed by living organisms. Of particular importance is the possibility to study these interactions at the very first steps of the reaction, i.e. at the molecular level.

The silicomolybdate spectrophotometric method offers a simple, widely applicable tool to gain such information. The similarity of the conclusions that could be drawn from the study of sodium silicate solutions using the yellow SMA method and the silicon cathecolate solution using the blue SMA reagent shows that they are suitable for the investigation of biopolymer-based biomimetic systems. In particular, the observed effect of proteins and polysaccharide nicely fits the hypothesis that arose from the study of natural systems.

However, this technique suffers from several limitations that would require complementary analytical approaches. For instance, it would be of high interest to be able to titrate oligomers larger than disilicic acid. A fluorescent probe to follow silica polymerisation was recently proposed but is not, at this time, specific enough towards the degree of oligomerization [42]. Alternatively, the low NMR-sensitivity of dilute solution may be overcome using ${ }^{29} \mathrm{Si}$-enriched silica precursors but this would be a costly approach. The presence of polymers can also prevent the use of chromatography methods that were previously successful in studying silicate solutions [43].

These remarks suggest that such silicate/biopolymers systems should also be studied from the point of view of the modification of the biopolymer structure and reactivity resulting from the presence of the mineral precursors. This approach would benefit from the most recent developments of protein characterization techniques such as NMR [44] and mass spectrometry [45], as well as the possibility to use time-resolved fluorescence technics [46], giving access to the dynamics of the reactions.

Nevertheless, when going from biopolymers to whole cells, the SMA method remains a method of choice to study the interactions between silica and living organisms. In fact, small silica molecular species are the more readily available silica source for such organisms [32], and the titration of these precursors is a specificity of this method.

\section{References}

[1] E. Dujardin and S. Mann, Adv. Mater. 14 (2002), 775.

[2] S. Weiner and L. Addadi, J. Mater. Chem. 7 (1997), 689.

[3] N. Kroger and M. Sumper, in: Biomineralization, from Biology to Biotechnology and Medical Application, E. Bauerlein, ed., Wiley-VCH, Weinheim, 2000, pp. 150-170.

[4] S. Mann, J. Mater. Chem. 5 (1995), 935.

[5] K. Schwarz and M. Epple, Chem. Eur. J. 4 (1998), 1898.

[6] J. Aizenberg, J. Cryst. Growth 211 (2000), 143.

[7] T.L. Simpson and B.E. Volcani, eds, Silicon and Siliceous Structures in Biological Systems, Springer-Verlag, New York, 1981.

[8] G.B. Alexander, J. Am. Chem. Soc. 75 (1953), 5655.

[9] F.E. Round, R.M. Crawford and D.G. Mann, The Diatoms, Cambridge University Press, Cambridge, 1990.

[10] T. Coradin, C. Roux and J. Livage, J. Phys.: Condens. Matter 13 (2001), R673.

[11] C.C. Perry, in: Biomineralisation, Chemical and Biological Perspectives, S. Mann, J. Webb and R.J.P. Williams, eds, VCH, Weinheim, 1989, pp. 223-256. 
[12] R.K. Iler, The Chemistry of Silica, Wiley-Interscience, New York, 1979.

[13] A. Jolles and F. Neurath, Angew. Chem. 11 (1898), 893.

[14] J.D.H. Strickland, J. Am. Chem. Soc. 74 (1952), 862.

[15] S. Lacroix and M. Labalade, Anal. Chim. Acta 3 (1949), 383.

[16] J.B. Mullin and J.P. Riley, Anal. Chim. Acta 12 (1955), 162.

[17] G.J.S. Govett, Anal. Chim. Acta 25 (1961), 69.

[18] V.W. Truesdale and C.J. Smith, Analyst 100 (1975), 203.

[19] M.L. Balconi, F. Sigon, M. Borgarello, R. Ferraroli and F. Realini, Anal. Chim. Acta 234 (1990), 167.

[20] A. Kamatani and O. Oku, Marine Chem. 68 (2000), 219.

[21] S. Ross and I.D. Morrison, Colloidal Systems and Interfaces, Wiley-Interscience, 1988.

[22] M. Feist, V.N. Molchanov, L.P. Kazanskii, E.A. Torchenkova and V.I. Spitsyn, Zh. Neorg. Khim. 25 (1980), 733.

[23] M. Coudurier, B. Baudru and J.B. Donnet, Bull. Soc. Chim. Fr. 9 (1971), 3147.

[24] J.H. Foulger, J. Am. Chem. Soc. 49 (1927), 429.

[25] L. Isaacs, Bull. Soc. Chim. Biol. 6 (1924), 157.

[26] V.W. Truesdale and C.J. Smith, Analyst 101 (1976), 19.

[27] J. Floch, S. Blain, D. Birot and P. Treguer, Anal. Chim. Acta 377 (1998), 157.

[28] C.C. Perry and T. Keeling-Tucker, J. Inorg. Biochem. 69 (1998), 181.

[29] M.C. Schwartz, Ind. Eng. Chem., Anal. Ed. 14 (1942), 893.

[30] C.C. Perry and T. Keeling-Tucker, J. Biol. Inorg. Chem. 5 (2000), 537.

[31] C.J. Brinker and G. Scherrer, The Physics and Chemistry of Sol-Gel Processing, Academic Press, Boston, 1990.

[32] T. Coradin and P.J. Lopez, ChemBioChem 4 (2003), 251.

[33] C.C. Harrison and N. Loton, J. Chem. Soc., Faraday Trans. 91 (1995), 4287.

[34] T. Coradin and J. Livage, Colloids Surf. B 21 (2001), 329.

[35] T. Coradin, A. Coupé and J. Livage, Mat. Res. Soc. Symp. Proc. 724 (2002), N7.20.1.

[36] T. Coradin, A. Coupé and J. Livage, Colloids Surf. B 29 (2003), 189.

[37] T. Mizutani, H. Nagase, N. Fujiwara and H. Ogoshi, Bull. Chem. Soc. Jpn. 71 (1998), 2017.

[38] T. Coradin, O. Durupthy and J. Livage, Langmuir 18 (2002), 2331.

[39] C.C. Perry and Y. Lu, J. Chem. Soc., Faraday Trans. 88 (1992), 2915.

[40] C.C. Perry and T. Keeling-Tucker, Chem. Commun. (1998), 2587.

[41] C.C. Perry and T. Keeling-Tucker, Colloid Polym. Sci. 281 (2003), 652.

[42] K. Shimizu, Y. Del Amo, M.A. Brzezinski, G.D. Stucky and D.E. Morse, Chem. Biol. 8 (2001), 1051.

[43] T. Tarutani, Anal. Sci. 5 (1989), 245.

[44] K. Wutrich, NMR of Proteins and Nucleic Acids, Wiley, New York, 1986.

[45] S.A. Trauger, W. Webb and G. Siuzdak, Spectroscopy 16 (2002), 15.

[46] J.R. Lakowicz, ed., Principles of Fluorescence Spectroscopy, Plenum Press, New York, 1983. 


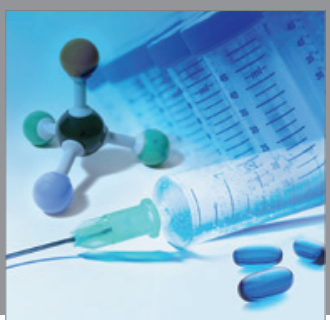

International Journal of

Medicinal Chemistry

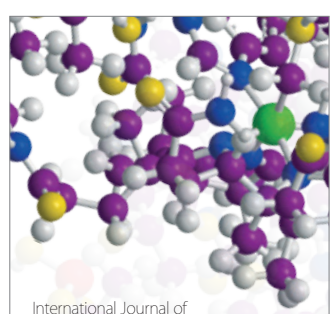

Carbohydrate Chemistry

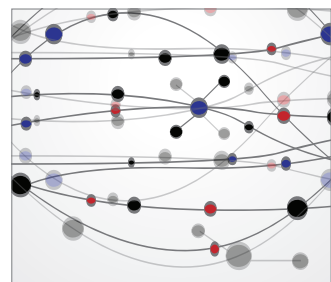

The Scientific World Journal
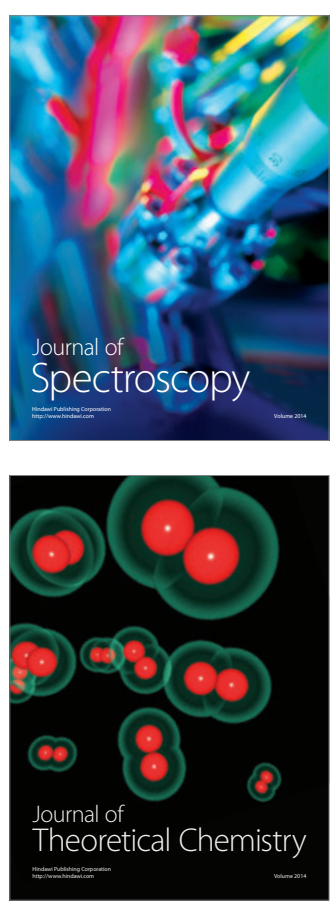
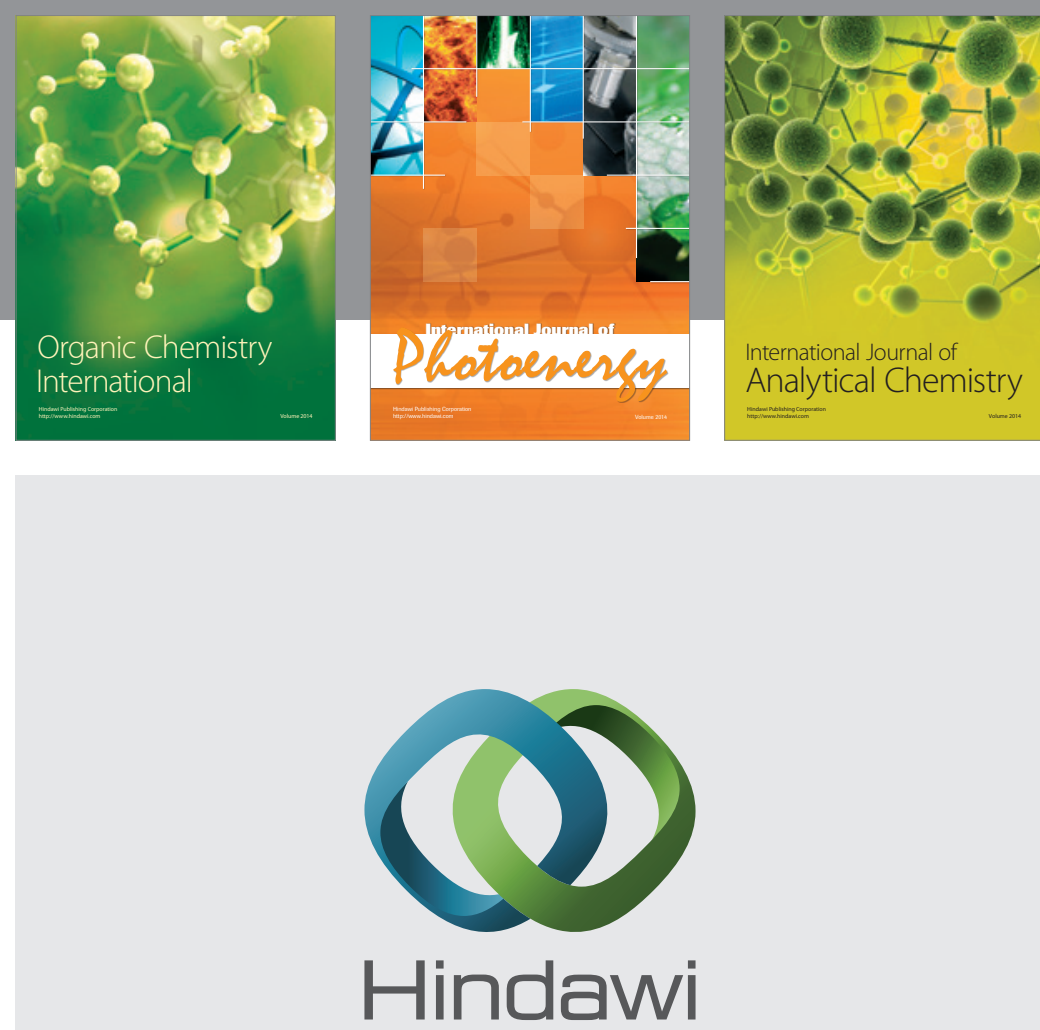

Submit your manuscripts at

http://www.hindawi.com
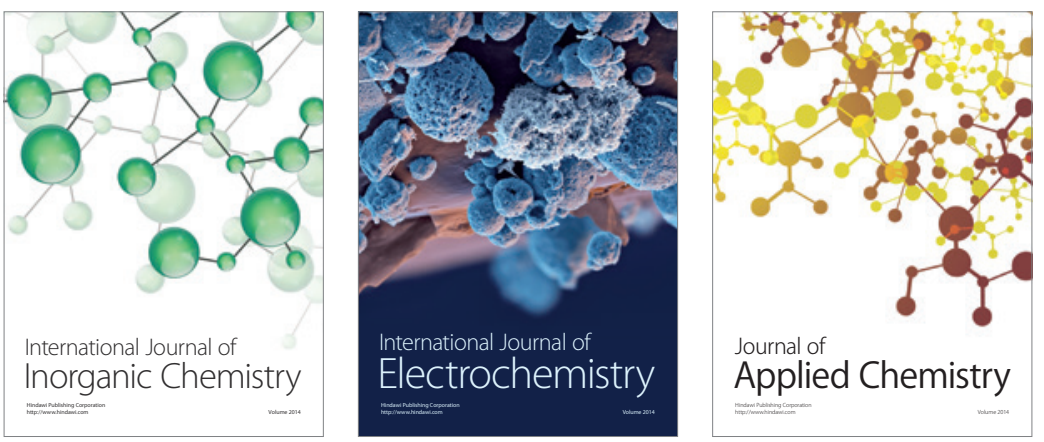

Journal of

Applied Chemistry
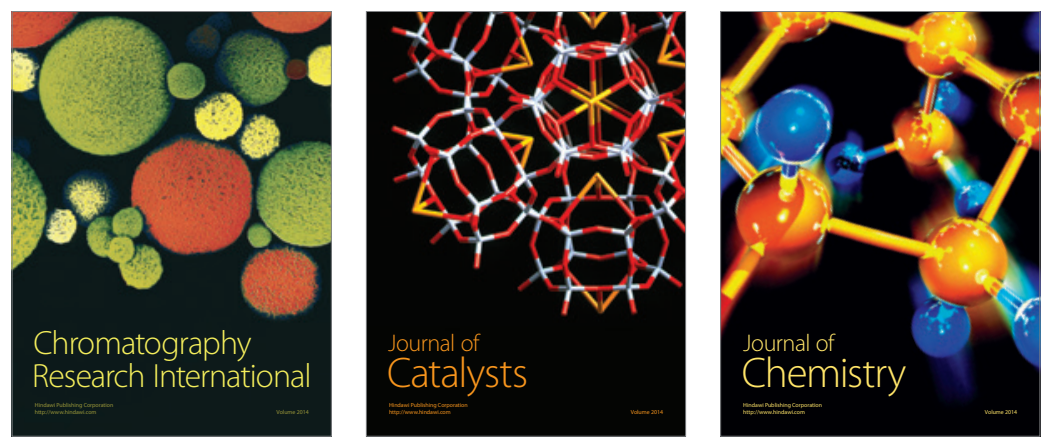
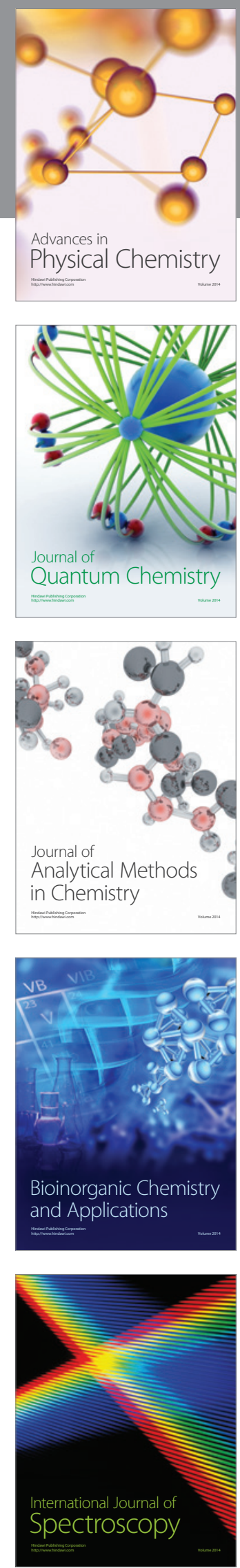